\title{
Out-of-Plane Focusing Grating Couplers for Silicon Photonics Integration With Optical MRAM Technology
}

\author{
Hanna Becker ${ }^{\circledR}$, Member, IEEE, Clemens J. Krückel $^{\circledR}$, Member, IEEE, Dries Van Thourhout ${ }^{\circledR}$, Member, IEEE, \\ and Martijn J. R. Heck ${ }^{\circledR}$, Member, IEEE
}

\begin{abstract}
We present the design methodology and experimental characterization of compact out-of-plane focusing grating couplers for integration with magnetoresistive random access memory technology. Focusing grating couplers have recently found attention as layer-couplers for photonic-electronic integration. The components we demonstrate are designed for a wavelength of $1550 \mathrm{~nm}$, fabricated in a standard $220 \mathrm{~nm}$ SOI photonic platform and optimized given the fabrication restrictions for standard 193-nm UV lithography. For the first time, we extend the design based on the phase matching condition to a two-dimensional (2-D) grating design with two optical input ports. We further present the experimental characterization of the focusing behaviour by spatially probing the emitted beam with a tapered-and-lensed fiber and demonstrate the polarization controlling capabilities of the 2-D FGCs.
\end{abstract}

Index Terms-FDTD, photonic-electronic integration, photonic integrated circuits, polarization, silicon photonics, vertical focusing grating couplers.

\section{INTRODUCTION}

$\mathbf{T}$ HE complexity and maturity of photonic integrated circuits (PICs) is steadily increasing and PICs are finding their way into more and more applications. One of these applications is alloptical or optically-enabled memory technology [1]-[3], which is currently still outperformed by high-performance electronic memories. Combining the high standards of electronic memory with the potential of fast and energy efficient PICs promises improved performance of all-electrical memory and adds memory functionalities to PICS by harvesting advantages of both platforms. Photonic-electronic integration in general has been advertised to enable new functionalities interconnecting both platforms, saving energy by reducing or eliminating receiver

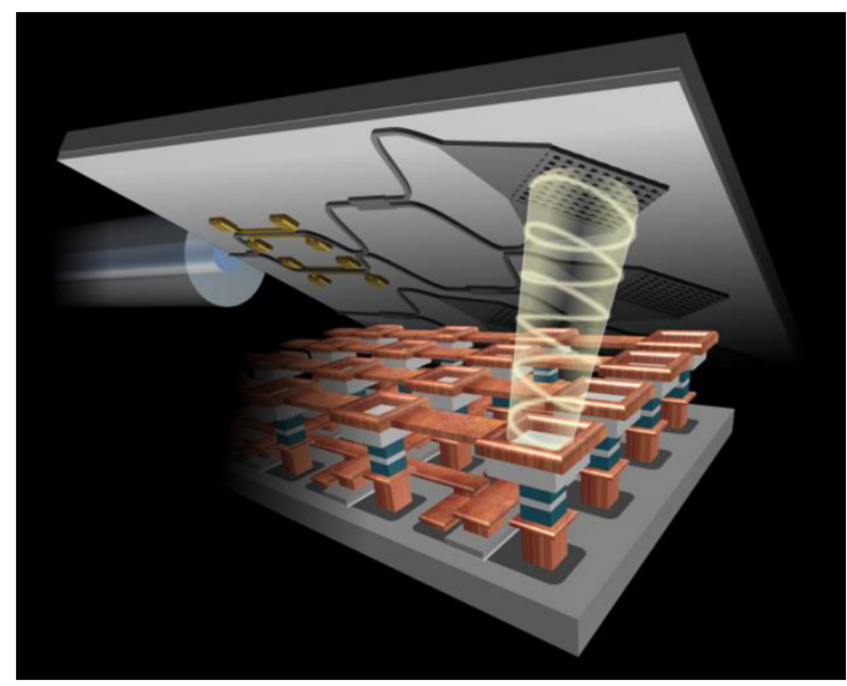

Fig. 1. Schematic of the photonic integration proposed by the EU SPICE project [5]. The photonic chip shown on the top couples circularly polarized light by means of a 2D FGC to the electronic chip with the individual MTJ memory elements displayed on the bottom.

circuit energies as well as by running large synchronous systems using PICs and improving speed when switching from electrons to photons [4]. The novel approach for optically-enabled magnetoresistive random access memory (MRAM) presented in the scope of the EU SPICE project [5] aims to integrate mature silicon PICs with all-optically switchable (AOS) materials [6] integrated in magnetic tunnel junctions (MTJs) [7]. The heterogeneous integration approach is illustrated in Fig. 1. Integration of the two platforms requires efficient optical coupling from the photonic to the electronic chip, which can deliver high optical intensities exceeding the threshold energy needed to switch the magnetic state of the memory element. Grating couplers are a suitable solution for such layer coupling components [4], [8].

Grating couplers are key-components in integrated photonics and are well-known for fiber-to-chip coupling [9]. Efficiencies have been pushed towards near unity values [10] and promise nearly loss-less coupling from waveguide to free-space or fiber. More recently, they have been increasingly designed to allow for more complex beam-shaping [11], free-space beam forming [12] and focusing [13]. 


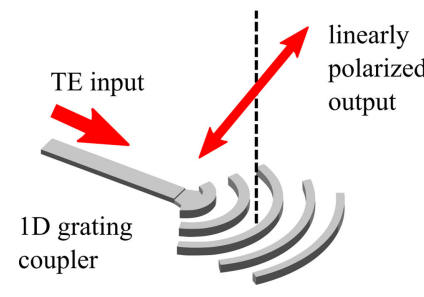

(a)

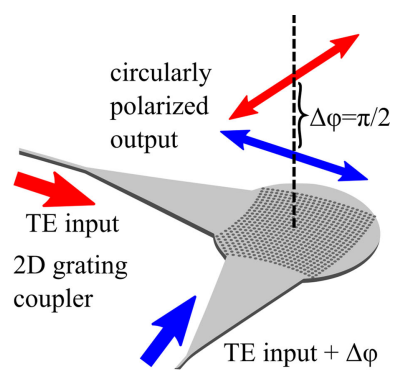

(b)
Fig. 2. Conceptional design idea for the (a) one-dimensional and the (b) twodimensional grating coupler.

In-plane focusing grating couplers (FGCs) [14] are nowadays frequently used in grating coupler designs as they reduce or even eliminate the need for long adiabatic tapers towards the grating area. Out-of-plane focusing grating couplers have also been around for some while as demonstrated by [15] for the read and write operation of optical-disk data storage. More recent examples were fabricated in the SiN platform for lab-on-chip applications using light in the visible wavelength regime demonstrating e.g., fluorescence detection or ion-trapping [16]-[18]. A design on a silicon-on-insulator platform for a long-workingdistance grating couplers is presented in [19]. The referenced designs have in common that they focus at comparatively large distances (focal length $>$ aperture) and at off-vertical emission angles. These design choices ensure decent efficiency by only slowly varying grating period and thus emission angle and by avoiding the high reflections associated with the vertical coupling condition [9].

We present the design methodology, simulation and experimental characterization of out-of-plane FGCs as layer couplers for photonic integration with AOS MRAM technology. The FGCs focus the light at a micrometer distance with near-vertical emission angle and small footprint with the design incentive to provide high optical pulse energies to surpass a given threshold value required for AOS of the magnetization and thus switching of the resistive state of the MTJ. This is achieved by focusing the emitted light as tightly as possible to increase the intensity within the sub-wavelength scaled MTJ [20]. The focusing characteristic was implemented in one- and two-dimensional grating coupler designs. The one-dimensional FGC (see Fig. 2.a) has one optical input waveguide, emitting linearly polarized light. For the twodimensional FGC (see Fig. 2.b), we expand the design principle and apply the phase matching condition to two optical input ports. Controlling the relative phase of the input ports allows for full polarization control of the emitted light and thus enables helicity dependent AOS [21].

\section{Compact Out-of-Plane Focusing Grating Couplers}

The design considerations we present in the following differ from the previously mentioned examples from literature, since we target near-vertical, high numerical aperture (NA) characteristics to meet the main objective of ensuring that the threshold value for AOS is exceeded. Additionally, we need to (a)

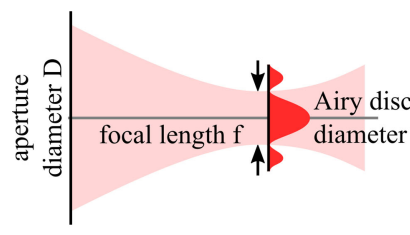

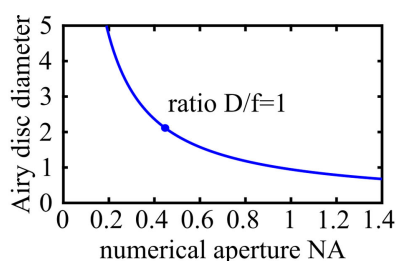

(b)

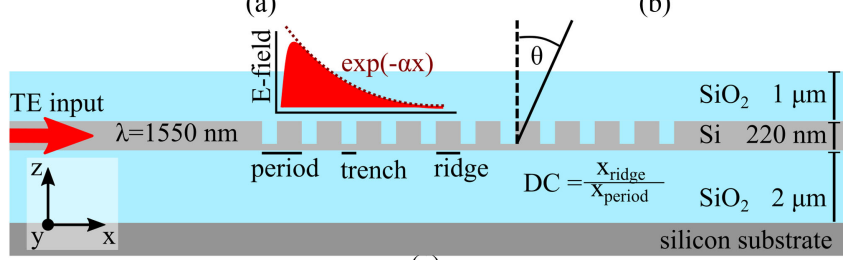

(c)

Fig. 3. (a) Geometric illustration of the involved parameters for focusing at focal length $f$ and aperture diameter $D$. (b) Illustration of the dependence of diffraction-limited focal spot size and NA with best trade-off at a ratio of one of aperture diameter over focal length $D / f=1$. (c) Schematic illustrating the grating parameters and platform dimensions.

consider the integration density requiring the components to have a small footprint. This poses the trade-off consideration of device footprint, NA, focal spot position and design restrictions in terms of single-diffraction order condition. Leading to FGCs with strongly varying grating periods over a short distance. On the one hand, this very discrete change of grating period is challenging in terms of the selection of the individual grating periods, since slightly offset periods can influence the performance dramatically. On the other hand, it enables us to avoid the very periodicity of exact vertical coupling and thus high back reflections. While very high efficiencies have been recorded for grating couplers in the past, these designs require one, or a combination of the following non-standard fabrication features: small feature sizes [22], adjusted substrate layers and custom etch depths [23]-[25], or additional fabrication processes to add metallic mirrors [26]. We optimize the presented FGCs given the commercial standards of a $220 \mathrm{~nm}$-SOI technology multi-project-wafer run [27] without additional post-processing needs, keeping the devices easily manufacturable.

\section{Design Methodology}

For the applicability of FGCs in photonic-electronic integration, we need to maintain a small footprint to allow for dense integration and to reduce overall footprint of the technology. Additionally we need to achieve high directionality values and tight focusing for efficient optical coupling between the platforms. Thus, we aim to design near-vertical diffraction-limited focusing layer-couplers with large NA, but restrict the grating footprint (aperture diameter $D$ ) to reasonable values.

This confronts us with a trade-off between small spot size and small footprint. The mentioned parameters are illustrated in Fig. 3.a and Fig. 3.b showing the relationship of the diffraction limited focal spot size (Airy disc diameter) of an imaging setup and its NA. We identify the sweet spot around an NA of $\approx$ 0.5 at a ratio of aperture diameter $D$ to focal length $f$ of 1 or larger. 


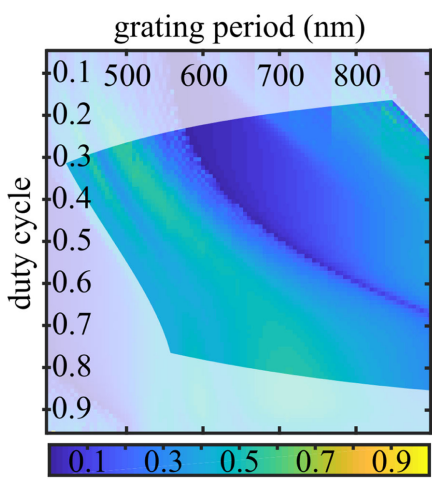

(a) Directionality

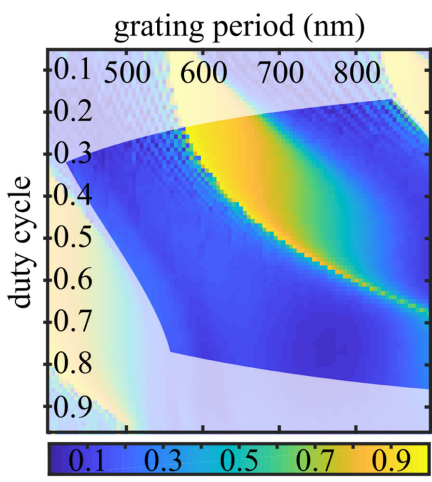

(b) Reflection

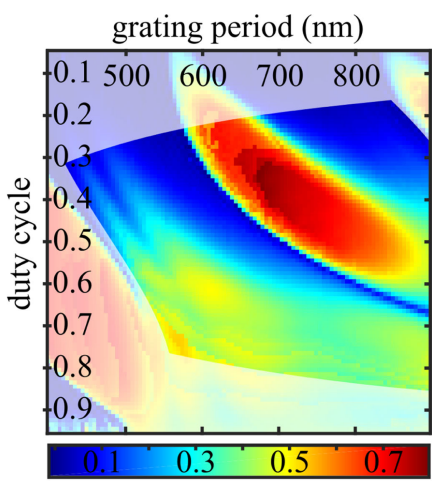

(c) Grating strength $\alpha$

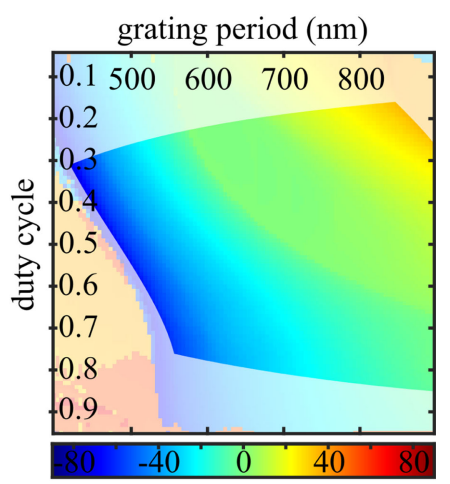

(d) Diffraction Angle $\theta$

Fig. 4. Simulation results at $1550 \mathrm{~nm}$ for the grating parameters as a function of grating period and DC for fully etched grooves in a $220 \mathrm{~nm}$ Si-layer on $2 \mu \mathrm{m}$ BOX oxide. Process restrictions and single order free-space diffraction restrict the parameter space as indicated by the greyed-out areas.

\section{A. ID Out-of-Plane Focusing Grating Couplers}

Focusing for both $1 \mathrm{D}$ and $2 \mathrm{D}$ designs is based on the phase matching condition (PMC) [15], which relies on calculating the phase difference between the incident wave from the waveguide and the output wave coupled by the grating. The PMC demands that diffracted waves from the center of each grating period interfere constructively at the focal point $F\left(x_{f}, y_{f}, z_{f}\right)$ above the grating

$$
\begin{aligned}
n_{c l} & \sqrt{x_{f}^{2}+y_{f}^{2}}+m_{1} \cdot \lambda_{c} \\
= & n_{\text {eff }} \sqrt{x^{2}+y^{2}}+n_{c l} \cdot \sqrt{\left(x_{f}-x\right)^{2}+\left(y_{f}-y\right)^{2}+z_{f}^{2}} .
\end{aligned}
$$

Equation (1) is numerically solved with the in-plane coordinates $(x, y)$ for optical path differences of integer multiples $m_{1}$ of the wavelength $\lambda_{c}$ in vacuum. Whereas the cladding refractive index is given by $n_{c l}$. The effective index $n_{e f f}$ of the grating region is approximated according to Rytov's formula [28]. Assuming we launch a single mode TE mode into the input of the grating coupler and define the duty cycle as $D C=x_{\text {ridge }} / x_{\text {period }}$ as illustrated in Fig. 3.c the effective index is approximated by

$$
n_{\text {eff }}^{2}=D C \cdot n_{\text {slab }}^{2}+(1-D C) \cdot n_{\text {etch }}^{2} .
$$

This uses the effective index of the slab waveguide $n_{\text {slab }}$ and the effective index of the grating trenches $n_{\text {etch }}$. For our designs we can use technology providing a $220 \mathrm{~nm}$ silicon layer and etch depths of $70 \mathrm{~nm}, 150 \mathrm{~nm}$ and $220 \mathrm{~nm}$. The effective indices for the slab waveguides needed for (1) are calculated in an FDTD simulation assuming a silicon oxide cladding with refractive in$\operatorname{dex} n_{S_{i 0_{2}}}=1.45$. The simulated values for the three etch depths are $n_{\text {eff }, 70 \mathrm{~nm}}=1.91, n_{\text {eff }, 150 \mathrm{~nm}}=2.54, n_{\text {eff }, 220 \mathrm{~nm}}=2.85$.

We numerically solve the PMC to get elliptically curved grating lines with chirped periodicity along the propagation direction. The elliptical grating lines have been demonstrated for in-plane focusing to the optical input port, to reduce or even eliminate the need for a tapered design [29]. In our case they additionally account for out-of-plane focusing in the orthogonal direction to the propagation direction. While chirp and periodicity determine the coupling angle $\theta$ and thus focusing along the propagation direction.
Equation (1) assumes one common effective index for the whole grating area, which is not the case, especially in our design where we get large variations of periodicity and DC. For the one-dimensional design, we try to counter this systematic error by generating look-up tables based on 2D FDTD simulations. We simulate the parameter space of periodicity and DC of periodic grating structures in the platform illustrated in Fig. 3.c. The advantage of these $2 \mathrm{D}$ simulations is, that the parameter sweeps can be calculated with reasonable time and computational effort and performance parameters are easily extracted. The parameters are displayed in Fig. 4.a-d as functions of duty cycle and grating period. Directionality $T_{u p}=P_{u p} / P_{i n}$ and reflection $R=P_{R} / P_{i n}$ are both expressed as optical power ratios compared to input power $P_{i n}$ incident at the waveguide port. The diffraction angle $\theta$ is directly accessible from the simulation, while the grating strength $\alpha$ is calculated from fitting an exponential decaying electric field magnitude $E$ along the length $x$ of the uniform grating

$$
E(x) \propto \exp (-\alpha \cdot x) .
$$

Apodization of the grating strength along the grating allows to shape the mode field above the grating. It additionally improves the transition from waveguide to grating area resulting in reduced back-reflections and increased directionality. To achieve a Gaussian output profile $G(x)$ the grating strength should be varied according to [30]

$$
2 \alpha=G^{2}(x) /\left(1-\int_{0}^{x} G^{2}(x) d x\right) .
$$

We show the exemplary grating strength profile for a $10 \mu \mathrm{m}$ grating in Fig. 5.a. Based on this profile and the chosen focal spot, we select suitable period and DC values from the look-up table finding the best fit for periodicity, coupling angle and grating strength while preferring combinations with better directionality. The periodicity along the grating varies from $520 \mathrm{~nm}$ to $865 \mathrm{~nm}$ and the duty cycle ranges from 0.36 to 0.93 for the shown example. Fig. 5.b shows the focusing behaviour based on the ray-tracing method from the center of the period given the coupling angle of the selected period and DC. This method allows the implementation of an optimization routine within the 


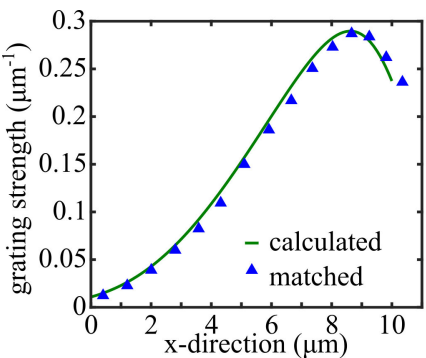

(a)

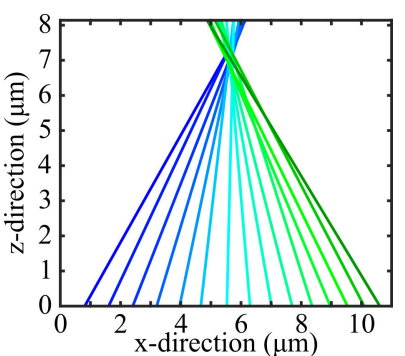

(b)
Fig. 5. (a) Calculated and matched grating strength values for Gaussian output field profile. (b) Beam tracing method of the assembled grating given the coupling angle and DC from the simulated look-up tables from the center each grating period.

constraints of the fabrication limitations in terms of a minimum feature size of $130 \mathrm{~nm}$ and pre-determined silicon layer thickness and etch depths. The fabrication restrictions and the condition for single-order emission restrict the parameter space available for the grating design and is illustrated by the grayed-out areas in Fig. 4. The assembled grating was then simulated in a full 3D FDTD simulation environment and periodicity and duty cycle were optimized further in a brute force approach, but restricting variations to around the selected values. Simulation results yield efficiencies in terms of directionality above $40 \%$, which is typical performance for grating couplers in comparable technologies [31]. $21 \%$ of this upwards directed intensity are within the full $1 / e^{2}$ width of the beam. Improvements of directionality can be expected for different silicon layer thicknesses and smaller feature sizes [26]. A $70 \mathrm{~nm}$ poly-silicon layer in a second fabrication run promises high-efficiency grating couplers [31]. The additional layer allows high contrast and thus stronger grating structures. We apply the previously described design methodology for the dimensions provided in this platform. The simulation results for these high efficiency 1D FGCs show promising directionality of over $70 \%$.

One-dimensional grating couplers are by design very polarization sensitive and emit linearly polarized light. If we want to allow for polarization diverse gratings [29] or enable polarization control of the emitted light as necessary for helicity dependent AOS, the grating coupler needs to be designed for two input ports.

\section{B. 2D Out-of-Plane Focusing Grating Couplers}

The general design principle for 2D out-of-plane FGCs remains the PMC described in (1) but has to be expanded to also meet a second PMC for the second optical input port

$$
\begin{aligned}
n_{c l} & \sqrt{\left(x_{f}-x_{2}\right)^{2}+\left(y_{f}-y_{2}\right)^{2}}+m_{2} \cdot \lambda_{c} \\
& =n_{e f f} \sqrt{\left(x-x_{2}\right)^{2}+\left(y-y_{2}\right)^{2}} \\
& +n_{c l} \sqrt{\left(x_{f}-x\right)^{2}+z_{f}^{2}+\left(y_{f}-y\right)^{2}} .
\end{aligned}
$$

Given the second input, the second PMC can be expressed in terms of a coordinate rotation and displacement to the second

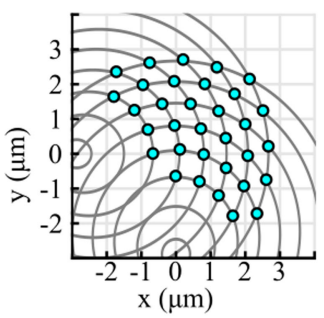

(a)

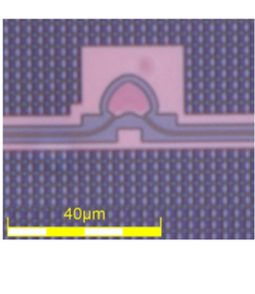

(b)

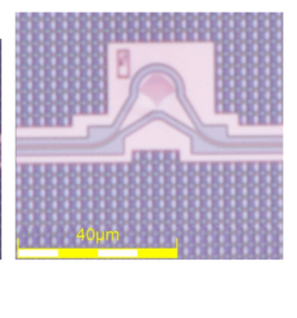

(c)
Fig. 6. (a) Schematic for the numerical solution to the two phase matching conditions (1) and (5). (b) Micrograph of taperless 2D FGC design. (c) Micrograph of tapered 2D FGC design with $10 \times 10 \mu \mathrm{m}$ grating area.

input port located at $\left(x_{2}, y_{2}\right)$. We design the grating for two optical quasi TE inputs with a relative angle of 90 degrees to one another. This should allow us to emit circularly polarized light by introducing a $\pm \pi / 2$-phase shift on one of the inputs.

Each PMC results in elliptical grating lines centered around their respective origin. Solutions to both conditions are the intersection of these ellipses and dictate the photonic crystal type hole-array of the grating. The numerical approach to get the solutions to the hole array is illustrated in Fig. 6.a. Our design effort converges towards a taperless design as shown in Fig. 6.b, which provides a small footprint of $10 \times 10 \mu \mathrm{m}$, but does not include adiabatic tapering. In this case the mode will expand in the grating area not using the full aperture area and thus not reaching NA values as designed. Other designs include short tapers into a grating area of $10 \times 10 \mu \mathrm{m}$ (Fig. 6.c). Designing the grating couplers for a compact $10 \times 10 \mu \mathrm{m}$ footprint has the advantage that the numerical solution can be implemented in a full 3D simulation environment with a reasonable simulation volume and thus adequate computational effort. This allows us to optimize and control the radius and etch depth of the hole-array within the FDTD environment. These parameters allow us to adjust the refractive index and grating strength to find the best performance based on the simulations. Since purely FDTD based optimization routines would still require extensive computation and time, we opt for a Matlab-driven optimization. In the first step the hole array is numerically solved and fed into the FDTD environment. We then optimize the hole diameter in a brute-force routine within the FDTD program by varying the value around the initial approximation to maximize the performance of the simulated device. The adjusted hole diameter can be used to adjust the initially assumed refractive index and thus the numerical solutions providing a new hole-array layout which again can be implemented into the FDTD optimization routine. From the simulations the grating performance parameters like coupling efficiency, FWHM of the focal spot and the simulated focal spot position can be extracted to evaluate the design. For the 2D FGCs we did not include apodization of the grating strength as we have for the 1D case. With the intention to reduce back-reflections by transitioning from low to high contrast grating structures, we combined the hole-array solutions of the different effective indices of shallowly etched holes and fully etched holes. The first row was implemented using the shallow-etch solution and the rest of the grating used the hole-array of the fully-etched 
solution. Micrographs of two fabricated devices of the above mentioned designs are shown in Fig. 6.b and c.

Another advantage of the compact designs is, that they all yield a broad bandwidth with the $3 \mathrm{~dB}$ bandwidth generally exceeding the simulation widow of $100 \mathrm{~nm}$. This is desirable as it allows for the coupling of short optical pulses, needed for all-optical switching. The described design methodology was applied to a multitude of FGCs with varying focal spot distances, ranging from 3-7 $\mu \mathrm{m}$. We evaluate our design approach in a first step by measuring the focusing characteristics of the fabricated gratings.

\section{EXPERIMENTAL EVALUATION}

Previously presented FGCs have been evaluated by surface normal imaging systems [13], [18] or SNOM technology [16]. In this paper, we present a beam profile characterization by evaluating the spatial mode overlap coupling efficiency of grating to tapered-and-lensed fiber, which can be implemented on many silicon photonic chip test setups. Focusing is determined by tracking the beam profile's evolution normal to the silicon photonic chip's surface (in z-direction). The measurement is enabled by automatic three-axis stages that can be programmed to move at sub-micrometer accuracy. To account for near vertical coupling we mount a tapered-and-lensed fiber $\left(1=e^{2}\right.$ spot size $2.5 \mu \mathrm{m}$ ) vertically above the chip on one of the three-axis stages. The small mode-field area allows probing of beam profiles of similar dimensions in the $1-2 \mu \mathrm{m}$ diameter range. On the input, a standard single-mode fiber couples light from an external tunable laser source through a standard grating coupler and a straight ridge waveguide to the FGC under test. We program the stages to align the fibers to a defined coordinate above the chip and start the measurement by more precisely aligning the fibers to the $\mathrm{x}, \mathrm{y}$-coordinates of highest coupling efficiency. This position marks the center for a cross-hair sweep of the lensed-and-tapered fiber in the xy-plane, scanning the coupling efficiency spatially resolved in two orthogonal directions. The fiber position above the chip is increased stepwise to predefined z-coordinates, where the scan procedure is repeated for every vertical position. A set of measurement data for a one-dimensional grating coupler is shown in Fig. 7 as $\mathrm{x}$ - and $y$-scan. The measured traces are normalized to the maximum coupling efficiency of the measurement set. We can now fit these scan profiles to the mode overlap integral $\eta$ of an elliptical Gaussian mode profile $E_{\text {test }}$ and the fiber mode $E_{\text {fiber }}$ with * indicating the complex conjugate and integrals over the area $A$

$$
\eta(x, y)=\frac{\left|\int E_{\text {fiber }}(x, y) \cdot E_{\text {test }}^{*} d A\right|^{2}}{\int\left|E_{\text {fiber }}(x, y)\right|^{2} d A \cdot \int\left|E_{\text {test }}\right|^{2} d A} .
$$

The trace providing the best fit for an assumed $E_{\text {test }}$ mode profile lets us extract the beam width of that mode profile. In Fig. 7 the maximum coupling efficiency and the full-widthhalf-maximum (FWHM) values are plotted as a function of z-position. In this way, we can track the profile of the emitted beam by the FGC. The expected behaviour of a focused beam can be clearly observed, as the FWHM values show a minimum at the beam waist, while the coupling efficiency peaks near

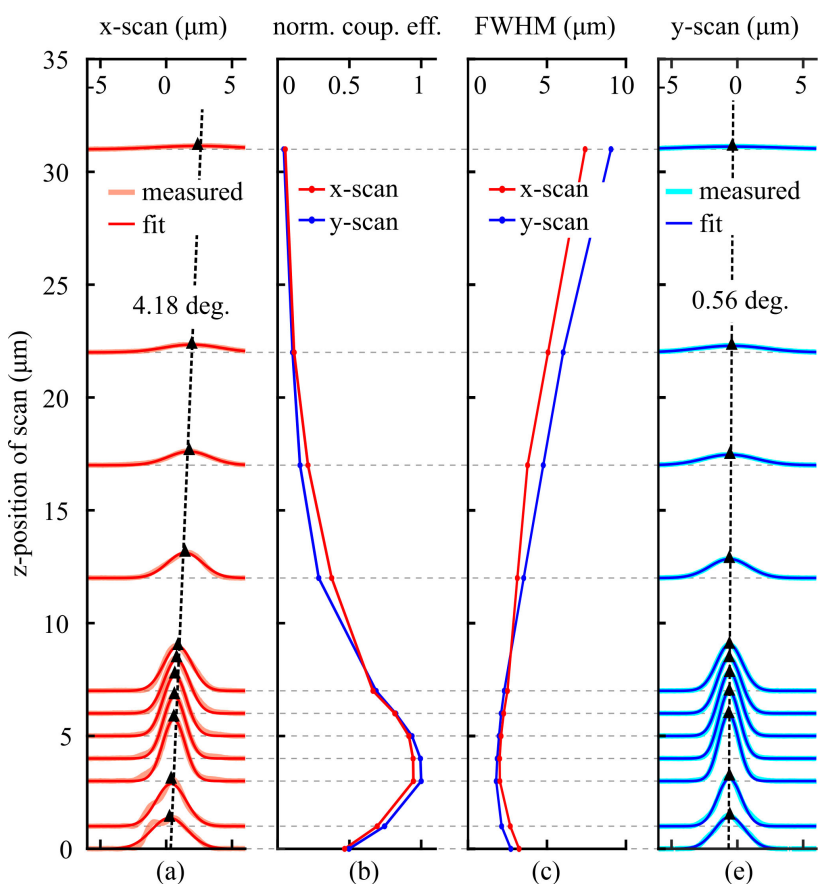

(c)

(e)

Fig. 7. Experimental evaluation of focusing characteristics at a wavelength of $1550 \mathrm{~nm}$. (a) Fiber coupling efficiency scan in x-direction. (b) Maximum measured coupling efficiency, normalized to the maximum value of the measurement set (FGC-to-fiber coupling efficiency -13.7 dB). (c) Estimated FWHM of emitted beam. (d) Fiber coupling efficiency scan in y-direction.

this focal spot. The exemplary shown measurements give an estimate of the FWHM in x- and y-direction of $2.3 \mu \mathrm{m}$ and $2.1 \mu \mathrm{m}$ and thus suggest a rather uniformly shaped, circular beam profile. Additionally, we can trace the peak coupling efficiencies of the measured traces along the beam's propagation direction and determine the overall coupling angle of the beam. For the example shown in Fig. 7, we find a coupling angle of 4.2 degrees off the vertical direction in $\mathrm{x}$-direction and an negligible off-axis emission angle of 0.6 degrees for the y-direction. The maximum measured grating-to-fiber coupling efficiency in the shown measurement set was $-13.7 \mathrm{~dB}$.

The measurement routine was performed for both $1 \mathrm{D}$ and $2 \mathrm{D}$ FGCs and confirms the validity of the design methodology to construct out-of-plane FGCs. The measured traces of the 2D FGCs can show slightly distorted focal spots, with differences between the FWHM values obtained from x- and y-scan. We can also see some astigmatism in the designs, where we find the narrowest beam waist at different $\mathrm{z}$-positions for $\mathrm{x}$ - and $y$-scan. Fig. 8 shows the extracted maximum coupling efficiencies and FWHM values of a 2D FGC showing a difference of the narrowest beam waist of the orthogonal scans of about $1 \mu \mathrm{m}$ in z-direction. The additional feature of the novel 2D out-of-plane FGCs presented here is the opportunity to control the polarization state of the emitted light, as will be discussed in the next section.

\section{A. Polarization Control}

To demonstrate the polarization controlling capabilities of the two-dimensional grating couplers, we included balanced 


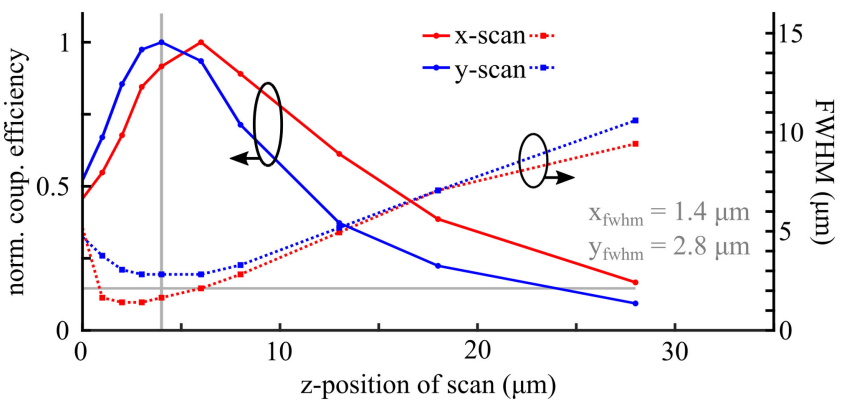

Fig. 8. Experimental evaluation of focusing characteristics of a 2D FGC presented by maximum measured coupling efficiency, normalized to the maximum value of the measurement set (FGC-to-fiber coupling efficiency $-19.8 \mathrm{~dB}$ ), and the calculated FWHM against z-position. The focal spot is indicated by the vertical grey line at $4 \mu \mathrm{m}$ where the product of FWHM from $\mathrm{x}$ - and $\mathrm{y}$-scan is minimum

Mach-Zehnder interferometer (MZI) type structures, splitting the incident light with $1 \times 2$ or $2 \times 2$ multimode interferometers (MMIs) and adding resistive heaters to thermally tune the relative phase of the two optical inputs to the 2D FGCs. The heaters were added to passives silicon chip in a back-end metallization process.

The emitted light is coupled into a vertically mounted polarisation maintaining fiber, to match the near-vertical emission of the grating couplers. We analyse the polarization state with a polarization controller/analyser based on cascaded liquid crystal based $\lambda / 2-, \lambda / 4$-waveplates and a linear polariser. Given the \pm 90 degrees rotation of the $\lambda / 4$-waveplates the polarization state is analysed based on the measured transmission curve as shown in Fig. 9.a. We match the transmission curve with a numerical least-square fit method to a polarization state defined by azimuth angle $\theta$ and elevation angle $\epsilon$. The calculated transmission curve uses Jones matrix polarization analysis [32].

The determined polarization states are illustrated on the normalized Poincaré sphere with the conventional notation $2 \theta$ for the angle of the linear polarization (main axis) and $2 \epsilon$ for the angle on Poincaré sphere describing the degree of ellipticity. The poles of the sphere indicate circularly polarized light at $2 \epsilon= \pm 90$ degrees. Measurements for different applied voltages to the phase shifter in the MZI controlled FGC are shown in Fig. 9.b. The full round-trip around the sphere indicates, as anticipated, that we can continuously change the state of polarization by tuning the relative phase delay of the two inputs.

Additionally to the MZI structures, we probe the emitted polarization states of the a ring-loaded MZI structure. Measurements are illustrated on the Poincaré sphere in Fig. 9.c together with the micrograph of one of the investigated structures. This second polarization control scheme relies on ring resonators. The measurements were obtained with a ring modulator with a free-spectral range of $14.2 \mathrm{~nm}$ and a resonance at $\lambda_{\text {res }}=1543.8 \mathrm{~nm}$ with FWHM of $0.6 \mathrm{~nm}$ using a directional coupler with cross-coupling coefficient of $\kappa=0.4$. The polarization state measurement allows us to extract the induced phase change by the modulator which is plotted in Fig. 9.d of the detuning $\phi$ together with the transmission spectrum of the ring. Our ring resonator exhibits a continuous phase shift of $2 \pi$, whereas a more abrupt phase change of $\pi$ can be observed crossing the resonance.

\section{DISCUSSION}

The design effort behind this work focused on implementing out-of-plane FGCs by utilizing the available foundry facilities for fabrication. Optimization was done within a simulation routine taking the given constraints into account. This approach highlights the potential to mass-manufacture these devices and integrate them on large-scale in mature and reliable fabrication processes, which is important to consider if a technology aims to be market ready. The choice to design the FGCs near-vertical focusing is driven by the integrability of the device. This demands a compact design that can still focus near the diffraction limit: for a compact design, we favour grating parameters with a large grating strength such that we can diffract the light out of the plane efficiently over a short distance. Yet, we have to work within the restrictions given by the fabrication process and the single-diffraction order condition as indicated in Fig. 4. We found the most promising and practical solutions to the PMC for near-vertical focusing, while off-vertical focusing would very quickly force the focal spot further away from the surface. We pursued this high-NA focusing, as this is potentially easier to align and integrate in the mentioned flip-chip approach.

The presented evaluation of the focusing characteristic and beam profiling offers a simple, fast approach for a first evaluation of coupling angle and mode profile of grating couplers in general. While the grating-to-fiber efficiency of $-13.7 \mathrm{~dB}$ might seem low, our main objective is not to couple to an external fiber, but to achieve high optical intensities in the focal spot, and thus the grating emission profile is not matched to the fiber mode. Accounting for the mode mismatch to the fitted elliptical Gaussian mode and the offset of emission angle and fiber angle gives a correction of at least $1 \mathrm{~dB}$. More importantly, it is not possible to assess a reliable directionality value, using the measurement technique explained in Section IV since we require a highly resolved electrical field profile for calculating the coupling efficiencies of these small spot sizes with the fiber mode. For an accurate estimation of the grating-to-fiber coupling efficiency, we require a more precise field profile than that given by the elliptical Gaussian profile approximation we used in Eq. 6 . High-NA surface normal imaging or scanning near-field optical microscopy (SNOM) measurements should give more insight into these performance parameters. The measurement results for the 1D FGCs indicate circular beam profiles and the beam waist at the same z-position for both orthogonal scan directions. Implying that the chosen effective index method works well in combination with the PMC and the look-up tables. In the case of the 2D FGC we see some astigmatism and more elliptical beam profiles. This is an indicator that relying on the phase matching conditions is adequate, but not entirely sufficient to produce a well-focused beam.

The 2D FGCs come with the advantage that they allow for polarization control of the emitted light when adding a relative phase shift between the inputs. As Ring resonators 


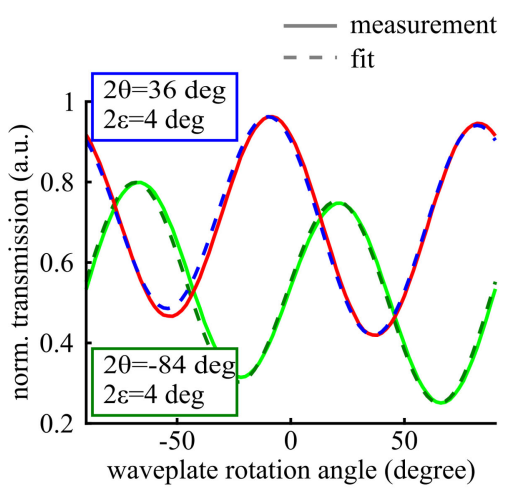

(a)

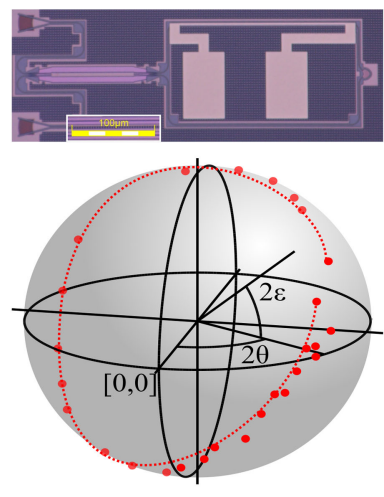

(b)

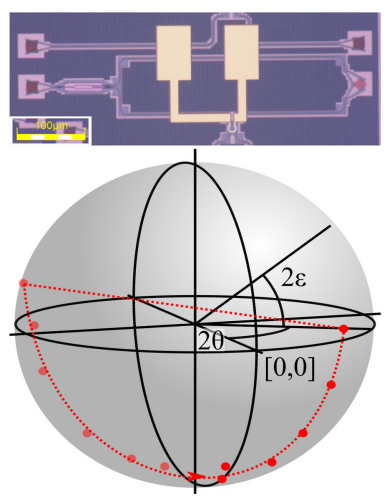

(c)

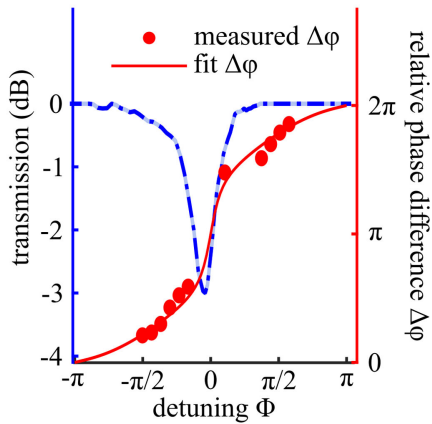

(d)

Fig. 9. Polarization control with FGC. (a) Two exemplary measurement traces and the calculated curves given the azimuth angle $2 \theta$ and elevation angle $2 \epsilon$. (b) Micrograph of the MZI structure with the thermal phase shifter on the upper arm and the polarization states measured with this configuration. (c) Micrograph of the ring-loaded MZI strucutre and the polarization states measured with this configuration.

exhibit an abrupt phase change for critical coupling [33], they pose as a promising path to fast and energy-efficient polarization state switching or for spatial mode multiplexing. The abrupt phase change of the ring-loaded MZI modulation scheme is of particular interest as this offers high-contrast polarization switching induced by a low-energy modulation scheme. Reference [34] shows that carrier-injection based ring modulators can potentially cut the consumed energy by half, compared to MZI modulation and offer switching energies below $3 \mathrm{pJ}$.

\section{CONCLUSION}

In conclusion, we were able to demonstrate compact $1 \mathrm{D}$ and 2D out-of-plane FGCs and verify their focusing properties by evaluating the spatial coupling efficiencies to a lensedand-tapered fiber. Additionally we were able to show that 2D grating couplers offer the additional feature of controlling the polarization state of the emitted light. The presented results are promising on the way forward to use these devices as photonic layer couplers and for photonic integration with optical MRAM technology.

Looking forward, the emission efficiency can be further improved considering fabrication processes that allow for stronger gratings like additional poly-silicon layers, multiple etch-depths and smaller feature sizes. This paves the way towards exceeding coupling efficiencies of $-3 \mathrm{~dB}$ making compact out-ofplane FGCs a suitable solution for photonic layer coupling and MTJ illumination in both heterogeneous and monolithic integration.

\section{ACKNOWLEDGMENT}

The authors would like to thank the Ghent University laboratory support staff for support on customizing and enabling the measurement setup, namely M. Vanslembrouck and J. Jans, and also S. Verstuyft for help with the post-process metallization of the passive silicon chips.

\section{REFERENCES}

[1] C. H. V. Agionas, "10 Gb/s optical random access memory (RAM) cell," Opt. Lett., vol. 44, no. 7, pp. 1821-1824, 2019.

[2] X. Li et al., "Fast and reliable storage using a 5 bit, nonvolatile photonic memory cell," Optica, vol. 6, no. 1, p. 1-6, 2019.

[3] M. Grajower, N. Mazurski, J. Shappir, and U. Levy, "Non-volatile silicon photonics using nanoscale flash memory technology," Laser Photon. Rev., vol. 12 , no. 4 , pp. 1-8, 2018.

[4] D. A. Miller, "Attojoule optoelectronics for low-energy information processing and communications," J. Lightw. Technol., vol. 35, no. 3, pp. 346396, Feb. 2017.

[5] "Spice project-Developing future memory," [Online]. Available: http:// spice-fetopen.eu/, Accessed on: Jun. 1, 2019.

[6] M. L. M. Lalieu, R. Lavrijsen, and B. Koopmans, "Integrating all-optical switching with spintronics," Nature Commun., vol. 10, no. 1, pp. 1-6, 2019.

[7] J.-Y. Chen, L. He, J.-P. Wang, and M. Li, "All-optical switching of magnetic tunnel junctions with single subpicosecond laser pulses," Phys. Rev. Appl., vol. 7, 2017, Art. no. 021001.

[8] N. Mangal, J. Missinne, G. Van Steenberge, J. Van Campenhout, and B. Snyder, "Performance evaluation of backside emitting O-band grating couplers for $100 \mu \mathrm{m}$-thick silicon photonics interposers," IEEE Photon. $J$. , vol. 11, no. 3, Jun. 2019, Art. no. 7101711.

[9] D. Taillaert et al., "Grating couplers for coupling between optical fibers and nanophotonic waveguides," Japanese J. Appl. Phys., vol. 45, no. 8 A, pp. 6071-6077, 2006.

[10] A. Michaels and E. Yablonovitch, "Inverse design of near unity efficiency perfectly vertical grating couplers," Opt. Express, vol. 26, no. 4, pp. 47664779, Feb. 2018

[11] C. T. Nadovich, W. D. Jemison, D. J. Kosciolek, and D. T. Crouse, "Focused apodized forked grating coupler," Opt. Express, vol. 25, no. 22, pp. $26861-26874$, Oct. 2017.

[12] J. Sun, E. Timurdogan, A. Yaacobi, E. S. Hosseini, and M. R. Watts, "Large-scale nanophotonic phased array," Nature, vol. 493, no. 7431, pp. 195-199, 2013.

[13] K. K. Mehta and R. J. Ram, "Precise and diffraction-limited waveguideto-free-space focusing gratings," Sci. Rep., vol. 7, no. 1, pp. 1-8, 2017.

[14] F. Van Laere et al., "Compact focusing grating couplers for silicon-oninsulator integrated circuits," IEEE Photon. Technol. Lett., vol. 19, no. 23, pp. 1919-1921, Dec. 2007.

[15] S. Ura, T. Suhara, H. Nishihara, and J. Koyama, "An integrated-optic disk pickup device," J. Lightw. Technol., vol. 4, no. 7, pp. 913-918, 1986.

[16] A. M. Katzenmeyer et al., "Volumetric imaging and characterization of focusing waveguide grating couplers," IEEE Photon. J., vol. 9, no. 6, Dec. 2017, Art. no. 2701009.

[17] K. K. Mehta et al., "Integrated optical addressing of an ion qubit," Nature Nanotechnol., vol. 11, no. 12, pp. 1066-1070, 2016.

[18] S. Kerman et al., "Integrated nanophotonic excitation and detection of fluorescent microparticles," ACS Photon., vol. 4, no. 8, pp. 1937-1944, 2017. 
[19] C. J. Oton, "Long-working-distance grating coupler for integrated optical devices," IEEE Photon. J., vol. 8, no. 1, Feb. 2016, Art. no. 2700208.

[20] M. Savoini et al., "Highly efficient all-optical switching of magnetization in GdFeCo microstructures by interference-enhanced absorption of light," Phys. Rev. B, Condens. Matter Mater. Phys., vol. 86, no. 14, pp. 1-5, 2012.

[21] K. Vahaplar et al., "All-optical magnetization reversal by circularly polarized laser pulses: Experiment and multiscale modeling," Phys. Rev. B, vol. 85 , no. 10, 2012, Art. no. 104402.

[22] J. Notaros et al., "Ultra-efficient CMOS fiber-to-chip grating couplers," in Proc. Opt. Fiber Commun. Conf., 2016, pp. 1-3.

[23] C. Li, H. Zhang, M. Yu, and G. Q. Lo, "CMOS-compatible high efficiency double-etched apodized waveguide grating coupler," Opt. Express, vol. 21, no. 7, 2013, Art. no. 7868

[24] Y. Ding, C. Peucheret, and H. Ou, "Ultra-high-efficiency apodized grating coupler using a fully etched photonic crystal," Opt. Lett., vol. 38, no. 15, pp. 2732-2734, 2013.

[25] X. Chen, C. Li, C. K. Y. Fung, S. M. G. Lo, and H. K. Tsang, "Apodized waveguide grating couplers for efficient coupling to optical fibers," IEEE Photon. Technol. Lett., vol. 22, no. 15, pp. 1156-1158, Aug. 2010.

[26] Y. Ding, C. Peucheret, H. Ou, and K. Yvind, "Fully etched apodized grating coupler on the SOI platform with $-0.58 \mathrm{~dB}$ coupling efficiency," Opt. Lett., vol. 39 , no. 18,2014 , Art. no. 5348 .

[27] "Europractice silicon photonics technologies," [Online]. Available: http:// europractice-ic.com/mpw-prototyping/siphotonics/imec/, Accessed on: Aug. 17, 2019.

[28] R. Halir et al., "Subwavelength-grating metamaterial structures for silicon photonic devices," Proc. IEEE, vol. 106, no. 12, pp. 2144-2157, Dec. 2018.

[29] F. Van Laere et al., "Focusing polarization diversity grating couplers in silicon-on-insulator," J. Lightw. Technol., vol. 27, no. 5, pp. 612-618, Mar. 2009.

[30] R. Marchetti et al., "High-efficiency grating-couplers: Demonstration of a new design strategy," Sci. Rep., vol. 7, no. 1, pp. 1-8, 2017.

[31] G. Roelkens, D. V. Thourhout, and R. Baets, "High efficiency silicon-oninsulator grating coupler based on a poly-silicon overlay," Opt. Express, vol. 14, no. 24, pp. 11622-11630, 2006.

[32] M. J. Padget and J. Courtial, "Poincaré-sphere equivalent for light beams containing orbital angular momentum," Opt. Lett., vol. 24, no. 7 , pp. 430-432, 1999.

[33] W. Bogaerts, P. D. Heyn, T. V. Vaerenbergh, K. D. Vos, and S Kumar, "Silicon microring resonators," Laser Photon. Rev., vol. 73, no. 1, pp. 47-73, 2012.

[34] C. J. Krückel, H. Becker, M. J. R. Heck, and D. V. Thourhout, "Towards high-speed energy-efficient pulse-switching networks implemented in carrier-injection-based Si-photonics," in Proc. Eur. Conf. Lasers ElectroOpt., Eur. Quantum Electron. Conf., Munich, 2019, p. 1.

Hanna Becker received the B.Sc. and M.Sc. degrees in physics from the University of Konstanz, Konstanz, Germany, in 2012 and 2015, respectively. She spent three months with the Marine Biological Laboratory (MBL), Woods Hole, USA, for a research stay for her bachelor's thesis on polarized fluorescence imaging. During her master's studies, she spent half a year in the electronics R\&D of Basler AG as an intern for quality assurance testing image quality and performing and programming firmware and functionality test. She returned to the University of Konstanz to work on a femtosecond-fiber laser system for her master thesis. In October 2016, she joined the Photonics Group, Aarhus University as a Ph.D. fellow to join the SPICE project and work on integrating silicon photonics with optical MRAM technology.
Clemens J. Krückel received the master's degree in electrical engineering from Technical University Hamburg, Harburg, Germany and the Ph.D. degree, for his work on nonlinear optics in silicon nitride waveguides, from the Chalmers University of Technology, Gothenburg, Sweden, in 2012 and 2017, respectively. $\mathrm{He}$ is currently a Postdoctoral Researcher with Ghent University-IMEC, Ghent, Belgium, where he is working toward reconfigurable optical networks within the SOI platform. His work is part of the European SPICE project that explores all-optical switching of magnetic memory cells. His research interests include nanofabrication technologies, carrier-dynamics of semiconductors, and integrated optoelectronics.

Dries Van Thourhout received the degree in physical engineering and the Ph.D. degree from Ghent University, Ghent, Belgium, in 1995 and 2000, respectively. From October 2000 to September 2002, he was with Lucent Technologies, Bell Laboratories, New Jersey, USA, working on the design, processing, and characterization of $\mathrm{InP} / \mathrm{InGaAsP}$ monolithically integrated devices. In October 2002, he joined the Department of Information Technology (INTEC), Ghent University, Belgium. He is currently a member of the permanent staff of the photonics group. Since 2008, he has a full time Professor. He is the Chair of the joint UGent-VUB Master in Photonics program and a Lecturer or Co-Lecturer for four courses within the Ghent University (Microphotonics, Advanced Photonics Laboratory, Photonic Semiconductor Components and Technology). He is coordinating the cleanroom activities of the research group and coordinating of the NAMIFAB centre of expertise. His research focuses on the design, fabrication, and characterization of integrated photonic devices. Main topics involve silicon nanophotonic devices and the integration of novel materials (III-V, graphene, ferro-electrics, quantum dots, etc.) on these waveguides to expand their functionality. He is working on applications for telecom, diatom, optical interconnect, and sensing.

Martijn J. R. Heck received the M.Sc. degree in applied physics and the Ph.D. degree in electrical engineering from the Eindhoven University of Technology, Eindhoven, The Netherlands, in 2002 and 2008, respectively. From 2007 to 2008, he was a Postdoctoral Researcher with the COBRA Research Institute, Eindhoven, where he was engaged in the development of a technology platform for active-passive integration of photonic integrated circuits. From 2008 to 2009, he was with the Laser Centre, Vrije Universiteit, Amsterdam, The Netherlands, where he was involved in the development of integrated frequency-combs generators. From 2009 to 2013, he was a Postdoctoral Researcher and an Associate Director with the Silicon Photonics Center, University of California, Santa Barbara, Santa Barbara, CA, USA, where he was involved in photonic integrated circuits based on the heterogeneous integration of silicon, silica, and III/V photonics. 